\title{
Ergonomic and usability analysis on a sample of automobile dashboards
}

\author{
Raíssa Carvalho and Marcelo Soares \\ Departament of Design, Federal University of Pernambuco, Av. Prof Moraes Rego,1235 Cidade Universitária, \\ PE-Brazil
}

\begin{abstract}
This is a research study based on an analysis which sets out to identify and pinpoint ergonomic and usability problems found in a sample of automobile dashboards. The sample consisted of three dashboards, of three different makes and characterized as being a popular model, an average model and a luxury model. The examination was conducted by observation, with the aid of photography, notes and open interview, questionnaires and performing tasks with users, the bases of which are on the principles laid down by methodologies. From this it was possible to point to the existence of problems such as: complaints about the layout, lighting, colors, available area, difficult access to points of interaction, such as buttons, and the difficult nomenclature of dials. Later, the findings and recommendations presented show the need for a further, deeper study, using more accurate tools, a larger sample of users, and an anthropometric study focused on the dashboard, since reading and understanding it have to be done quickly and accurately, and that more attention be given to the study of automobile dashboards, particularly in the most popular vehicles in order to maintain the standards of usability, and drivers' comfort and safety.
\end{abstract}

Keywords: ergonomics, panels usability, vehicle, cars, icons

\section{Introduction}

In the past, four decades ago, the organization of a system such as an automobile instrument panel posed no great challenge. The amount of information was limited as was its complexity, as a result of the low technology and number of functions, reduced the number of interactions with the panel.

Forty years later, the advance of technology has been remarkable. The increase in automobile functionalities and the requirements of the law itself has made the complexity expand, and increased the number of symbols and displays present on the panel. New icons have been inserted - these are not always easy to understand and, due to the fact of their being a novelty, very often different in their representation, so that they vary from make to make despite having the same goal, there being no standard.

As a result of this development, there is now a larger amount of information on the panel to be read, interpreted and processed using logical, effective and timely thinking. The little space it has and the large number of elements present have given rise to disorder.

Also with the advances in engineering and electronics, cars have become faster and the data need to be read, interpreted and responded to more quickly. The need has developed for a new study of the product, namely an ergonomic study of the system, through usability with a view to ensuring the viability and optimization of communication between the elements involved in the system of the automobile dashboard.

Since this is still a recurring need today, a study was made that addressed identifying the problems and applying the principles of ergonomics and usability based on the methodologies proposed by Moraes \& Mont'Alvão and by Jordan, based on a sample of three different models of dashboards, of different brands. The study examined issues such as postural, dimensional and instrumental problems, and sets out discussions and puts forward ergonomic recommendations.

\footnotetext{
*Corresponding author. E-mail: raissab_carvalho@hotmail.com
} 


\section{What is an instrument panel?}

The instrument panel is the part of the car responsible for the interaction between the driver and the vehicle, it is the device that outputs information on the system. It is the driver's point of access to the controls of the system. It is within that much of the human-machine interface for everyday use is summarised. Through it, the driver comes into contact with the data transmitted by the instruments in order to drive.

The panel consists of a set of indicators in the form of dials and displays, which exhibit quantities and values for each function performed. These are important in accomplishing the task of driving and can be exemplified as speed, engine temperature, rotations of the engine and giving warning of existing problems and the working of devices.

Regarding the content of the panel, different countries have different laws and specificities, However, most dials are common to many of them, such as the speedometer, the thermometer, the fuel level indicator, direction signal lights, and the warning light about oil and the battery.

Pieces of information passed through the automobile dashboard are mostly emitted by means of lumious signals and scales, helped by colors to make reading them easy.

\subsection{Dials and displays}

Dials or displays are devices that give information about the machine or the behavior of the system. They are set up as an interface from the moment in which they configure the exchange between man and machine (in this case, from the panel to the driver) (Iida, 2005).

According to Cushman \& Rosenberg (1991) displays are used to transmit information that cannot be directly known or easily understood. Good displays present information so that they can be read quickly and accurately interpreted.

To ensure the correct use of the dials, prior knowledge of their meaning is needed. Improper use of the dials could adversely affect the entire system by increasing the time the driver needs to understand the information and could induce him/her to error.

Dials are classified as (Iida, 2005):

- Qualitative: when they show values close to real ones, though not precise and thus only indicate the correct functioning, or otherwise, of the instrument.
- Quantitative: they present values linked to variables, usually with some reference number, such as the speedometer has.

And are presented in the forms:

- Analog: a scale plus a pointe, where one is fixed and the other mobile, and shows values that change rapidly and often.

- Digital: although they do not detect the changes as easily as analog ones, they are used when there is need for precision and quick and accurate reading.

Currently it is becoming easier to find digital displays, or electronic, displays in newer cars. However, in most cases this is just a different form of presentation, because the real value, inside the vehicle, is still obtained in analog form.

\subsection{Icons and symbols}

According to Peirce (apud in Bock et al, 1993) there are three types of signs: the symbol, the index and the icon.

The icon is the image that has some similarity with the object. It is a representative simplification of the object.

The symbol is an agreed image arising from ideas produced at a convention. Certain symbols sometimes do not refer directly to what they represent, but are part of an international classification, which causes them to become known by at least $66 \%$ of the population, which classifies them as a universal standard.

These signs are widely used for facilitating the reading and interpretation of some information, in lieu of words. Thus, responses are quicker and more effective.

The signs used in dashboards are considered international. Some of them are determined by a country's traffic code, as in the case of Brazil, which lays down requirements for locating, identifying and lighting of controls, indicators and lamps, thus providing greater security based on a set minimum level and standard.

\subsection{Colors in automobiles}

After World War II, the automobile increasingly became the populations' preferred mode of transportation. And in a short space of time, the manufacturers of paints and varnishes stopped placing limits on the use of colors when painting them.

However, since colors influence human perception, some safety problems began to appear in traffic, very often resulting in hazardous situations. 
The use of colors, however, was not confined to the exterior of the car. Colors started to be used in the panels. They were used in varying shades and intensities. Some like red, orange and blue were even laid down by law to represent certain icons on the panel, such as red for the warning indicator light, brake and battery, orange for the light indicating oil and blue for the indicator lights of headlights.

And, thus as in painting, difficulties also arose related to the use of these variants on the panel. The number of colors became extensive and not standardized and presenting them in the same icon, or icons of similar functions, is not always displayed uniformly or in agreement.

These problems, which linked to the interpretation of information through colors, are more an aggravating factor than ones that hinder proper reading and interpretation, just like the assimilation of the intensity and urgency of the data which is being transmitted by the panel.

\section{Methodologies used}

In the study, two methods were used for analysis. The first was related to ergonomic assessment, and used the SHTM, proposed by Anamaria Moraes and Cláudia Mont'Alvão and Claudia Moraes (2005). The second, which was intended to assess usability in the dashboards, was proposed by Patrick Jordan (1998).

Appraisal of the system was used to define the problem to be tackled. And to do so, due to the impossibility of using a system entirely, because of the enormous number of elements it has, use was made of a sample, thus limiting the number, getting close to reality and serving as the basis for the whole.

The sample was designed using three models: a basic model, represented by the Peugeot 206; an average type model, represented by the Honda Civic; and a deluxe model, represented by the Mitsubishi Pajero Full.

During the ergonomic appreciation, the following were carried out: modeling the system, problematization - in which the ergonomic dysfunctions (problems to do with posture, instruments, dimensions, information, actions, cognition, interaction, action movements were addressed as were accessibility, biology, accidents and operational, organizational, instructional and psychosocial ones), the dysfunctions SHTM man (psychoneuro and physiological problems) and dysfunctions of the SHTM machine (resistance and producibility problems and socio- cultural and semiological ones) - a table of the impact of problems presented and, subsequently, the ergonomic opinion and recommendations.

The evaluation of usability was about identifying the presence of this in the panels, using the principles proposed by Jordan's methodology.

The principles evaluated, in their degree of existence or their complete absence, were: consistency, compatibility, user features available, feedback, prevention and recovery from errors; the user's control, visual clarity, prioritization of functionality and information, technology transfer and the explicability of the system.

\section{Ergonomic analysis of the automobile dashboards}

The instrument panel is a set of indicators, the function of which is to give facts and data about the vehicle being driven and the state in which it is.

As seen, a panel has numerous dials and signs, and with the addition of technology, this number tends to increase. The number of pieces of existing information is at a very high level. This requires the driver to be knowledgeable and able to assimilate information about all the components and their meanings for the proper functioning of the system.

And so that the reading is made correctly and in good time, it is necessary that these are arranged ergonomically, with bases in usability, with the aim of enhancing the process.

The study focused on analysing usability in a sample of automobile dashboards. Choosing this topic came about due to seeing the need for a good arrangement, following an ergonomic standard that puts usability center-stage in the task to be performed.

Problems such as layout, use of the chromatic scale, the positioning of the icons, their size, type and spacing between them and of the sources used are some of the problems also evaluated together with the available area, the distance between the dashboard and the driver, visibility through the steering wheel and reflectivity of light on the dashboard.

\subsection{Peugeot 206-popular model (Figure 1)}

During the ergonomic analysis of the model, some problems were found, and classified according to the consequences for the system:

- Serious Impact: instrument problems (reflection of light, preventing the display of certain indicators), 
cognitive problems (the existence of new symbols that do not have a close relationship with what they represent, so that their meanings are ignored) and instructional problems (manual for the panel is complex as to explaining the symbols and their nomenclature);

- Medium impact: problems of movement (the hands of the dials cover the corresponding number, making it difficult to read), of posture (line of sight of the panel is affected by the limitation on seat adjustment and steering) and of informational (proximity of icons of similar representation cause the risk of a misreading);

- Mild impact: problems of interaction (access to the headlight adjustment) of resistance (the panel suffers with direct physical aggression and is prone to chemical corrosion), of biology (environment in which it is located is prone to dirt accumulating, both in its protective covering and on its base), of interaction (text messages that appear are time consuming and long), of dimensions (distribution of symbols and dials on the panel is not suitable) and of perception (lack of proximity to the arrows indicating direction with one's hands).

\subsection{Honda Civic-average model (Figure 2)}

During the ergonomic analysis of the model, found some problems were found and classified according to the consequences for the system:

- Severe Impact: information problems (information provided in a foreign language) and instructional problems (lack of clarity in explaining the functions and meanings of the signs of the panel);

- Medium impact: problems of viewing (some signs are obscured by the illumination of the neighboring sign), of movement (there are a large number of levers and handles for the reactions to the information provided by the panel), of perception (although there is a sound signal, it is not perceived due to its low volume), of instruments (little space for distributing the signs on the panel, which hinders the dissociation of the images) and of dimensions (discre

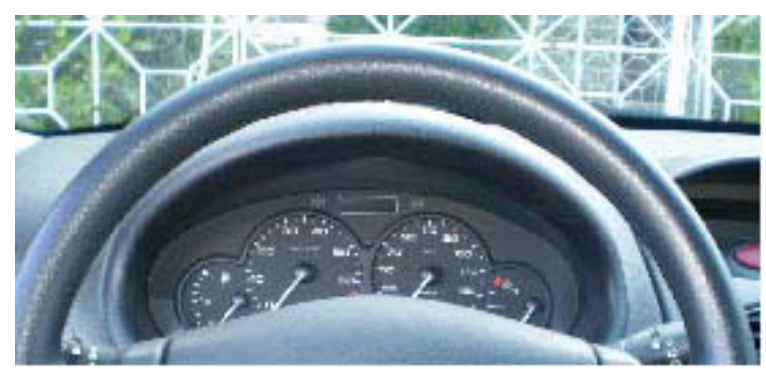

Figure 1 - Dashboard of Peugeot 206

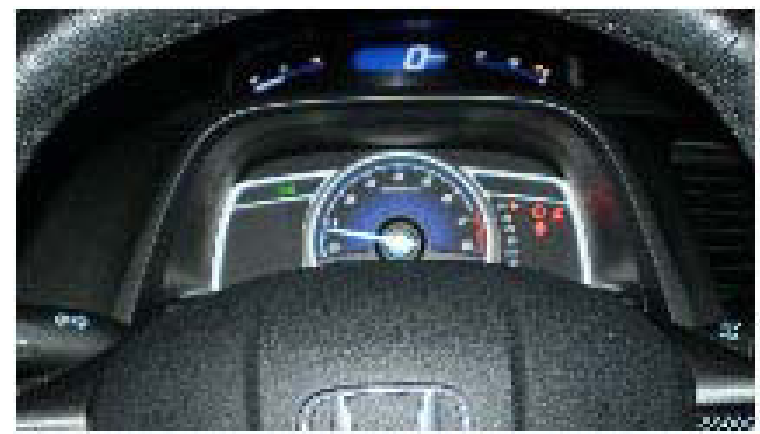

Figure 2 - Dashboard of Honda Civic

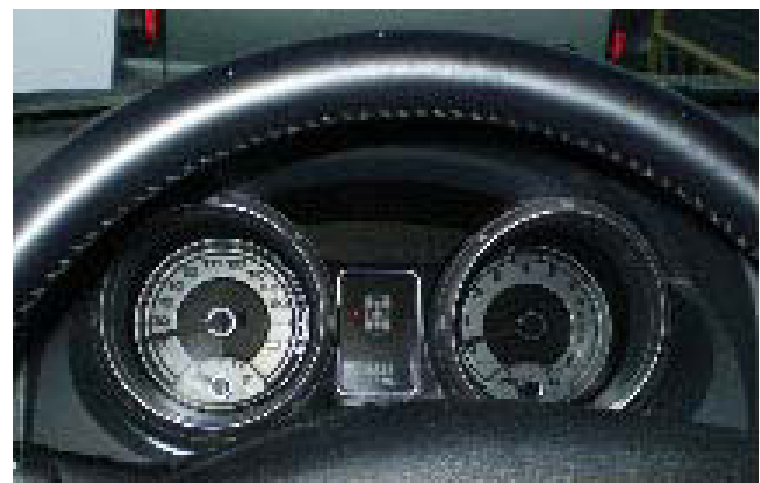

Figure 3 - Dashboard of Mitsubishi Pajero

pancy in prioritizing the functions as to the size in which they are presented);

- Mild impact: problems of instruments (the extra pieces of information passed on by the mileage meter is quite confusing for the user) of resistance (panel suffers with direct physical aggression and is prone to chemical corrosion), of biology (environment in which it is located is conducive to the accumulation of dirt, both in its protective covers and in its base), of interaction (the captions on the handles are very similar which confuses the driver), of dimensions (the small size of the mileage meter makes reading it difficult), of cognition (insertion of new icons that have no direct association with their meaning makes them hard to read, and the use of extremely similar signs to relate different functions) and of psychoneurophysics (the indicator of the level of very low fuel is triggered too early, and disturbs the driver). 


\subsection{Mitsubishi Pajero Full-deluxe model (Figure 3)}

After the ergonomic analysis of the model, some problems were found and classified according to the consequences for the system:

- Serious impact: semiological problems (due to the large number of new signs and captions it is necessary to have full knowledge of all of them and of the manual to manage to drive the car satisfactorily) and instrumental problems (little space for the luminous panel signs);

- Medium impact: problems of instruments (there was a bad choice of prioritizing the application of the dials), of dimensions (the area set aside for the panel is quite small), of viewing ("heavy" and dark chromatic choice, it is tiring to the eye and difficult to read), of interaction (the appearance of new buttons for new technologies, with their captions which are difficult to understand), of movement (the pointers of the largest dials cover the numbers indicated and of the smallest dials do not get close enough to a dis- tance that makes them easy to read) and of perception (the frames of the analog displays are very large and hinder viewing the other dials and signs);

- Mild impact: problems of cognition (insertion of several new signs which most users are not used to), of resistance (panel suffers with direct physical aggression and is prone to chemical corrosion), of biology (environment in which it is subject to the accumulation of dirt, both in its protective covering and at its base), and of instruction (appearance of items present also in other models, but in this one they differ in their representation).

\subsection{Ergonomic comparison between models}

With the result of the problems encountered in each of the models, a comparison was made between each of the categories of the problems in the three vehicles. The comparison aimed to point up which of the categories of each of them most urgently needs to be restudied (see Table 1).

Table 1

Ergonomic comparison between models

\begin{tabular}{|l|l|l|l|}
\hline Problem/Ca & Peugeot 206 & Honda Civic & Mitsubishi Pajero Full \\
\hline Postural & Medium impact & Mild impact & Mild impact \\
\hline Instrumental & Serious impact & Serious impact & Serious impact \\
\hline Dimensional & Mild impact & Medium impact & Medium impact \\
\hline Informational & Medium impact & Serious impact & Mild impact \\
\hline Visual & Mild impact & Medium impact & Medium impact \\
\hline Perceptual & Mild impact & Medium impact & Medium impact \\
\hline Cognitive & Serious impact & Serious impact & Mild impact \\
\hline Interacional & Serious impact & Mild impact & Medium impact \\
\hline Movement & Medium impact & Medium impact & Medium impact \\
\hline Biological & Mild impact & Mild impac & Mild impact \\
\hline Instructional & Serious impact & Serious impact & Mild impact \\
\hline Psychoneurophysical & Mild impact & Mild impact & Mild impact \\
\hline Of resistance & Mild impact & Mild impact & Mild impact \\
\hline Semiological & Mild impact & Mild impact & Serious impact \\
\hline
\end{tabular}

\section{Evaluating the usability of the automobile dashboards}

The usability evaluation conducted was qualitative in nature, using only four volunteers. Its objectives were to observe the main characteristics of the panel used by the user; to observe how a user views the panel; to observe the level of user satisfaction with the panels shown to them; to observe the user's opinion about the panels shown to them; and to draft an introduction about the study of usability in automobile dashboards.
The evaluation was based on analysing tasks and questionnaires on the users, on the dashboards in general, on the tasks performed and on the usability present in each model.

The questionnaires and tests were conducted in a period of three days and a total of 34 questionnaires distributed, of which two were two pilots and all of them answered. The sample of users was classified as their being in the 20 to 29 age range, two men and two women and all of them had a driver's license.

\subsection{Questionnaires on the users}

The questionnaire aimed to determine the experience that users had in the task of driving a vehicle 
and the knowledge they possessed in relation to the vehicle as a whole.

It was found that the users had average experience and that they all preferred hatch-back cars. And as to the frequency of day-to-day use of the vehicle it was found there was constant use for various activities.

\subsection{Questionnaire on instrument panels in general}

The purpose of this was to evaluate users' experience specifically with the instrument panel.

Questions of a general nature were asked to seek existing difficulties in relation to reading the dashboard and various situations in which it exhibited a piece of information which the driver found he/ she could not decipher.

They were also asked questions about what instruments they considered more important in the dashboard and which ones most called their attention. The responses were similar, and always stressed the speedometer, the fuel gauge, thermometer, brake lights and battery.

\subsection{Analysis of tasks}

This consisted of carrying out various timed tasks in order to determine the ease or difficulty users had in performing them. The tasks were evaluations of simple activities arising from daily use such as turning on the direction indicators, identifying the symbol for oil and the battery, reading the milometer, and so forth.

First contact with the vehicles caused difficulty for some users which was much greater than for others. However, in certain tasks, the difficulty proved to be very serious for everyone, regardless of the make of vehicle, such as the task of resetting the mileage meter.

The reasons for this were diverse, some because they do not know what the word means, even when they know where the display is, someone else as he/she really does not know what the mileage meter was, and the remainder because they did not manage to locate the reset miles device.

And as a result of this task, it was found that despite the existing experience and users declaring that they are familiar with the dashboard, when conducting the tasks, the process becomes a little more complicated.

\subsection{Questionnaires of tasks}

These were applied to assess the level of difficulty of the tasks performed in the vehicles. And while doing this, users' observations about these were collected.

The result was that, although some of them did not manage to perform the activities set, they did not consider the work was an action that was medium or difficult to do, and emphasized the lack of knowledge and the difficulty of reading and interpreting the dashboard.

\subsection{Comparison of usability between models}

From the usability evaluations obtained from the questionnaires and analysis, a comparison was made between some points of interaction seen in the models in order to grade the level of usability found in each (see Table 2).

\section{Ergonomic and usability recommendations}

As a result of the studies conducted, it may be said that the automobile dashboard is far from ideal. Ergonomic and usability problems are common to all vehicles, regardless of the category into which it is fited.

There is a lack of greater standardization, mainly by the signs related to the new technologies used, to how they are represented and to the space reserved for them. The lighting, the colors and the sources used also require study. 
Table 2

Comparison of usability between models

\begin{tabular}{|c|c|c|c|}
\hline Characteristics/car & Peugeot 206 & Honda Civic & Mitsubishi Pajero Full \\
\hline $\begin{array}{l}\text { Satisfaction as to the display } \\
\text { of information for the tasks } \\
\text { performed }\end{array}$ & Average & Good & Good \\
\hline $\begin{array}{l}\text { Satisfaction as to the amount } \\
\text { of information presented in } \\
\text { the panel }\end{array}$ & Average & Good & Good \\
\hline $\begin{array}{l}\text { Satisfaction as to the organ- } \\
\text { ization of the car dashboard } \\
\text { (company, distribution, etc) }\end{array}$ & Good & Good & Average \\
\hline $\begin{array}{l}\text { Quality lighting and color } \\
\text { panel }\end{array}$ & Good & Good & Good \\
\hline $\begin{array}{l}\text { Similarity of the panel in } \\
\text { performing the tasks de- } \\
\text { manded }\end{array}$ & Average & Average & Average \\
\hline $\begin{array}{l}\text { Similarity of the panel com- } \\
\text { pared to other panels that } \\
\text { already has some contact }\end{array}$ & Average & Poor & Poor \\
\hline $\begin{array}{l}\text { Effort related to the number } \\
\text { of human channels used in } \\
\text { carrying out human tasks }\end{array}$ & Average & Average & Poor \\
\hline $\begin{array}{l}\text { Quality of responses, issued } \\
\text { by the panel,to the tasks } \\
\text { performed }\end{array}$ & Average & Good & Good \\
\hline $\begin{array}{l}\text { Ease of correction and re- } \\
\text { covery from errors }\end{array}$ & Average & Good & Average \\
\hline $\begin{array}{l}\text { Quality control of the user in } \\
\text { performing the tasks }\end{array}$ & Average & Average & Average \\
\hline $\begin{array}{l}\text { Quality of the priority of } \\
\text { information in the panel }\end{array}$ & Good & Good & Good \\
\hline $\begin{array}{l}\text { Similarity of the technology } \\
\text { of the panel with others who } \\
\text { already have some contact }\end{array}$ & Average & Poor & Average \\
\hline $\begin{array}{l}\text { Quality of the explanation of } \\
\text { information by the car dash- } \\
\text { board }\end{array}$ & Average & Average & Good \\
\hline
\end{tabular}

\subsection{Ergonomic recommendations}

With regard to the previous recommendations for solving the problems found, it is possible to point to some general ones such as:

- Standardizing the icons in their format, color, function and name;

- Making it possible to adjust the brightness of the panel, since its intensity is linked to external luminosity;

- A better use in locating the action buttons used in response to information from the panel, especially the one that zeroes the mileage meter;

- A new way of passing on important information about the car, such as the check-up period, changing oil, etc. other than via the mileage meter, which causes confusion; perhaps using an on-board computer is the solution;
- The requirement for a minimum space between icons, other dials and limit areas, and

- Use of better displays taking into account studies already carried out.

\subsection{Recommendations of usability}

With regard to the previous recommendations for solving the problems found, it is possible to point to some general ones such as:

- The similarity existing between the sign of the oil indicator and that of electronic fuel injection makes it easy to confuse them, with some users not even considering that there had been a change in the representation of electronic fuel injection (they still took it in as a reference to the injection and not the engine, currently used ), and therefore there is a need to rethink its modes of exhibition;

- The display of some signs as the warning mode varies from car to car and can vary between being shown by the symbol of the triangle or the activation 
of the two directional arrows, which confuses the driver who will not be sure whether the alert was triggered correctly. Standardization is necessary;

- Signs like that of the battery and oil do not reflect their exact functions, which makes it difficult to understand what they mean, and also needs to be rethought; and,

- The presentations of the icons of oil and temperature should be reconsidered as they are easily confused because they show indications of liquids.

\section{Conclusion}

The study tackled the points described and necessary for a basic analysis on ergonomics and usability in automobile dashboards.

The ergonomic assessment addressed the system to be worked on and the problem with regard to the panels was defined. The tasks performed were reviewed as were the pieces of information passed to the driver and his expected reactions.

The evaluation of usability addressed the principles and characteristics of the panel, by analyzing them and testing them.

And having tackled the reviews, surveys, interviews, questionnaires and tests, the results were written down, described and analyzed, thus bringing the study to a close. Problems were pointed out in the areas of posture, information, actions, cognition, communication, to do with their being inherent, perceptual, and their clarity, efficiency, etc. As were possible solutions within the appropriate recommendations.

However, despite the research pointing to the existing difficulties, it does evaluate them more deeply, which would happen in an ergonomic diagnosis. This will fall to a further study.

In this study, directions are given and key issues highlighted, thus making these a basis for further work. It is for the next step to consider the analysis and to propose answers to the questions posed, by translating them into a better formulation of the automobile instrument panel.

\section{References}

[1] A. Banks and T. Fraser, O Guia Completo da Cor, São Paulo, 2007.

[2] W. Cushman and D. Rosenberg, Human Factors in Product Design, Amstedã, 1991.

[3] I. Iida. Ergonomia: Projeto e Producão, São Paulo, 2005.

[4] P. Jordan. An Introduction to Usability, London, 1998.

[5] N. Lefleur. Usability Evaluation: usability of iPod touch. Florida, 2010.

[6] A. Moraes and C.Mont'Alvão. Ergonomia: Conceitos e Aplicações, Rio de Janeiro, 2010.

[7] D. Norman. O Design do Dia-a-Dia, Rio de Janeiro, 1988.

[8] M.M. Soares. Ergonomics and design: a user-centred design method, In: Cyberg 2005. 